\title{
Inhibition of return and manual pointing movements
}

\author{
MARTIN H. FISCHER \\ University of Dundee, Dundee, Scotland \\ JAY PRATT \\ University of Toronto, Toronto, Ontario, Canada \\ and \\ SEBASTIAAN F. W. NEGGERS \\ University of Utrecht, Utrecht, The Netherlands
}

\begin{abstract}
To examine whether the motor inhibition of return (IOR) postulated by Taylor and Klein $(1998,2000)$ generalizes to manual guided movements or is restricted to saccadic responses, the following three experiments were conducted. The first experiment combined peripheral cues (which generate IOR) with four types of manual responses made to central targets (central arrow indicating the response location). The responses were made on a touch-screen and were the equivalent of either a detection keypress, a choice keypress, a detection-guided pointing movement, or a choice-guided pointing movement. No IOR was found for any of the responses. The second experiment replicated the main result under eye fixation control. In Experiment 3, peripheral cues and peripheral targets were used, and IOR was present in all responses. Overall, these findings suggest that motor-based IOR is restricted to the oculomotor system. Implications for motor-based IOR and attention-based IOR are discussed.
\end{abstract}

To better understand the role of visual-spatial attention for vision and for the control of action, researchers present visual cues, followed by targets, and record the response latencies of their observers. In one seminal study of this kind, Posner and Cohen (1984) reported that manual detection responses to peripheral target onsets could be either facilitated or inhibited by the prior presentation of peripheral cues. Replicating earlier work, they found that detection responses were shorter when the responserelevant target appeared at the cued location than when it appeared at the uncued location, provided that the time between the onsets of the cue and the target (stimulus onset asynchrony, or SOA) was less than $300 \mathrm{msec}$. Unexpectedly, they also found the reverse pattern of results, with target detection being slower at the cued location when the SOA was greater than $300 \mathrm{msec}$. This later occurring inhibitory effect of cuing was termed inhibition of return (IOR).

Preliminary results of this study were reported at the 41 st Annual Meeting of the Psychonomic Society (New Orleans, November 2000). This study was conducted while the first author was at the University of Munich. It was supported by an equipment fund from the Max Planck Institute for Psychological Research (Munich) to M.H.F. and NSERC grants to J.P. We thank Birgitt Aßfalg for data collection and Wolfgang Prinz for enabling this collaboration. Adrian von Muehlenen, Raymond Klein, Jason Ivanoff, and several anonymous reviewers provided helpful comments on previous drafts. Address correspondence to M. H. Fischer, Department of Psychology, University of Dundee, Dundee DD1 4HN, Scotland (e-mail: m.h.fischer@dundee.ac.uk).
The traditional view of IOR is that longer reaction times (RTs) to targets at cued than at to uncued locations reflect the output of a mechanism that inhibits attention from returning to previously attended locations (e.g., Maylor \& Hockey, 1985; Posner \& Cohen, 1984; Tipper, Driver, \& Weaver, 1991). An alternative view has recently been championed by Klein and Taylor (1994; Taylor \& Klein, 1998, 2000; see also Rafal, Calabresi, Brennan, \& Sciolto, 1989), in which the mechanism underlying IOR is motor, not attentional, in nature. This view suggests that IOR reflects a motor bias against responding to stimuli that occur at, or near, locations to which an eye movement has been programmed. Thus, it is possible to separate these alternative viewpoints into an attentional theory and a motor theory of IOR.

In conceptualizing the motor theory of IOR, Taylor and Klein (1998) examined what types of cues might generate IOR and what types of responses might reveal the inhibitory effect. ${ }^{1}$ On the basis of the literature, they suggested that any peripheral cue, regardless of whether it is responded to with an eye or a manual response, or not responded to at all, will generate IOR, because the abrupt appearance of a peripheral cue will automatically generate eye movement programming to the location of the cue. Central cues are also capable of generating IOR, but only if an eye movement is planned or executed to the location indicated by the central cue. This view, first suggested by Rafal et al. (1989) in their study in which peripheral and central cues were used, implicates oculomotor activity as the critical component in generating 
IOR. By consequence, this view also suggests that IOR will not be generated with manual responses, or no responses, to central cues.

Taylor and Klein (1998) also specified what types of responses should be sensitive in revealing IOR. The motor view suggests that because there is a motor bias against responding to a location, the initiation of either manual keypress or saccadic eye movement responses to peripheral targets will be inhibited. In addition, saccades made in response to a central target will be inhibited, because such a response is also made to a specific (inhibited) spatial location (see, also, Abrams \& Dobkin, 1994). A manual keypress response to a central target will not be inhibited, however, because it can be made with the information at the fixation location, and no information from (inhibited) peripheral locations is required. Thus, in order to show IOR, the cue must generate IOR, and the response must be sensitive to IOR.

These predictions were recently tested by Taylor and Klein (2000). Of the 24 conditions they reported in the paper, 2 are particularly important in the present context. Both of the 2 conditions used peripheral cues (which were not responded to), followed by central arrow targets. In one condition, a choice keypress (left or right index finger) was made in response to the central arrow target. This condition failed to show IOR. In the other condition, a saccadic eye movement was made to a peripheral box indicated by the central arrow (left or right box), and this experiment showed IOR. It is worth noting that neither of these conditions or results was new; they had previously been reported by Abrams and Dobkin (1994; see, also, Abrams \& Pratt, 2000). However, Abrams and colleagues were interested specifically in the effect of IOR in the oculomotor system. In the context of Taylor and Klein's (1998) proposal and the other conditions reported by Taylor and Klein (2000), the above findings suggest that there is a dissociation between visual-processing IOR (which may be due to inhibited attentional reorienting) and motor IOR (the slowing of directed motor responses).

In discussing the dissociation between visual-processing IOR and motor IOR, Taylor and Klein (2000) noted that several critical questions remain regarding the nature of IOR. One such question is whether or not manual pointing movements made in response to central targets will show IOR. As Taylor and Klein (2000) suggested, "the answer to this ... question is fundamental to the issue of whether the saccadic system really is the determinant of the type of inhibition that is observed or whether any spatially directed response may also produce a general motor inhibition" (p. 1653). In other words, if IOR is found with manual pointing responses and central targets, motor IOR is not limited to the oculomotor system but must be a more general form of motor inhibition. However, if IOR was not found with manual pointing responses and central targets, motor IOR may indeed be limited to the oculomotor system. The examination of various types of manual responses (keypress and point- ing) to both central and peripheral targets with regard to IOR was the focus of the present study.

Eye and hand movements differ in a number of waysfor example, with regard to their goals (information acquisition vs. object manipulation) and their motor-planning demands (cf. Fischer, 1997, p. 21, for details). However, there are reasons to expect that motor IOR will be found with central targets and manual pointing responses. First, as was noted by Bekkering, Pratt, and Abrams (1996), pointing movements share many features with saccadic eye movements. Importantly, both responses are spatially localized; they require spatial information in order to be completed. Indeed, Bekkering et al. found evidence that the gap effect, previously thought to be limited to saccadic responses, produces robust effects on manual pointing responses. Second, both saccades and manual pointing involve the superior colliculus (SC; e.g., Stuphorn, Hoffmann, \& Miller, 1999; Werner, 1993). The SC has also been implicated by Taylor and Klein (1998, 2000) as the likely locus of motor IOR. Indeed, there is now direct evidence that IOR is reflected at the singleunit level in the SC (Dorris, Klein, Everling, \& Munoz, 2002) and is not found when there is damage to the SC (Sapir, Soroker, Berger, \& Henik, 1999). Thus, one can expect that guided pointing movements will show IOR with central targets in the same manner that saccadic eye movements do.

The present study consists of three experiments. In the first experiment, peripheral no-response cues were followed by central targets that required various types of manual responses. These responses included two types of keypress responses and two types of guided pointing responses. As was found by Taylor and Klein (2000), the two keypress conditions did not show IOR with the central targets. More important, IOR was also not found with either type of manual pointing response. The finding of no IOR with central targets and pointing responses was replicated in Experiment 2, which also used eye movement monitoring for fixation control. In the third experiment, the same cues and responses were used as in the first experiment, but with peripheral targets. IOR was obtained in all conditions. In conjunction with previous studies, the present results suggest that motor IOR may be limited to the oculomotor system.

\section{EXPERIMENT 1}

This experiment combined peripheral no-response cues with central targets, the same cue-response pairing that did not yield IOR for Taylor and Klein (2000). However, unlike in Taylor and Klein (2000), four different types of manual responses were used, rather than just choice keypresses. For one response (simple touch, or ST), the participant was required to touch one predetermined peripheral response location upon the appearance of the central arrow target, irrespective of its direction. Essentially, ST was a simple detection response, involv- 
ing only one hand. For another response (choice touch, or CT), the participants were required to touch one of two peripheral response locations, depending on the direction of the arrow target. This response involved selecting between two hands and was the same type of response as that used by Taylor and Klein (2000). In both the ST and the CT responses, the participants placed their hand(s) above the peripheral response location(s), so no actual transport of the responding hand was required. In contrast, for the last two response conditions, the responding hand started at a central location and moved to a peripheral location to make the response. In the simple movement (SM) response, the participants moved to the same peripheral response location regardless of which direction the central target arrow indicated. This was essentially a target detection task with an aimed limb movement. For the final response (choice movement, or CM), the participants were required to make an aimed limb movement to the peripheral location indicated by the central arrow target.

As was mentioned earlier, finding IOR with the manual pointing responses would suggest that motor IOR is general and affects most spatially localized motoric responses, whereas not finding IOR with manual pointing responses would suggest that motor IOR affects only eye movements. Following Abrams and Dobkin (1994) and Taylor and Klein (2000), IOR was not expected to be found with either type of keypress response. In addition to an examination of RTs, the SM and CM responses also allowed for the examination of the effect of IOR on the response itself. This was done by examining the movement times (MTs) for responses to cued and uncued locations.

\section{Method}

Participants. Fifteen members from the human participants pool of the Psychology Department at the University of Munich participated in the experiment, including one of the authors. Eight participants were female. Two participants were left-handed; all others were right-handed according to self-report. Their ages ranged from 22 to 40 years (average, 31 years). All the participants had normal or corrected vision. The naive participants received DM $12 / \mathrm{h}$.

Apparatus. The participants were run individually in a quiet laboratory room with a dim background light. They were seated on a height-adjustable office chair in front of a Philips 4 CM 2299 Autoscan Professional Color monitor with 20-in. diagonal screen size. The screen was tilted at a $45^{\circ}$ angle and was equipped with a permanent touch interface (ELO-Touch, controlled with ELO-Graphics MonitorMouse 2.0). The spatial resolution of this touch interface corresponded to the pixel resolution of the screen, which was set to 1,024 horizontal $\times 768$ vertical pixels, respectively ( 26 pixels correspond to $10 \mathrm{~mm}$ ). An Apple 4400/200 PowerMac controlled stimulus presentation and response collection. No head restraint was used, to ensure natural movements. Responses were made with the index finger directly on the screen, and eye movements were not monitored.

The experimental display (see Figure 1) contained three rectangles $(10 \times 32 \mathrm{~mm}$ side length): A central start box and two peripheral response boxes that were outlined with a white frame (1 pixel) on a black background throughout the experiment. The distance between each response box and the start box (side to side) was $70 \mathrm{~mm}$. With an average viewing distance of $50 \mathrm{~cm}$, this display extended across $10.2^{\circ}$ of visual angle. The display was centered on the screen and remained visible throughout the experiment. Cues were implemented by coloring the corresponding boxes in red, targets by presenting a green arrow $(10 \times 5 \mathrm{~mm})$ in the top portion of the central box.

Procedure. Upon contact, the central start box changed from green to black to indicate each trial onset. A peripheral red cue appeared $1 \mathrm{sec}$ later for $200 \mathrm{msec}$ in one of the two lateralized response boxes, followed, after a $200-\mathrm{msec}$ delay interval, by a second central red cue $200 \mathrm{msec}$ in duration. Finally, after another 200-msec delay period, a green arrow target appeared at the fixation location in the experimental trials, whereas no target appeared in the catch trials. Thus, the SOA between peripheral cue and central target was $800 \mathrm{msec}$. The final display remained visible until either a touchdown had been registered or the maximum trial duration of 1,200 msec since onset of the central cue had elapsed. The participants were instructed to remain fixated on the central start box for the duration of each trial. Anticipatory responses (lift-offs or touchdowns prior to target onset), procrastinations (no response within 1,200 $\mathrm{msec}$ ), and touchdowns outside the correct response area (plus a 10-pixel tolerance range) led to corresponding feedback messages on the screen. These error trials were not repeated and were not used in the analyses.

Design. There were nine blocks of different response conditions in the experiment, all of which required touch responses on the computer screen. Consider first the SM and CM responses involving hand movements to a target. With the SM response, the participants moved either the left or the right index finger to a preassigned response box upon presentation of the central arrow target. For example, in a left-response block, an arrow pointing to either the left or the right box would always require a goal-directed movement to the left box. The SM responses were assessed in four conditions by crossing responding hand (left or right) with preassigned location of the response (left or right). With the CM response, the participants had to move the index finger from the start box to the response box indicated by the central arrow target. In two separate blocks, they used either the left or the right hand.

Consider now the responses involving touchdowns but no hand transport toward the response boxes. With ST and CT responses, the participants held their index finger(s) above one or both of the peripheral boxes, respectively. With the ST response, the participants responded with either the left or the right index finger on a preassigned side, irrespective of the direction of the arrow target. There were two blocks for the ST task, one each for left- and rightside touchdowns, always using the hand ipsilateral to the relevant response box. Finally, with the CT response the participants responded with the index finger signaled by the arrow (i.e., a flexion of the left index finger for a left-pointing arrow). This response required response selection between the left and the right hands in each trial. There was only one block of the CT task.

The experiment consisted of a total of 450 trials. For each of the nine blocks (two of ST, one of CT, four of SM, two of CM), there were 40 test trials and 10 catch trials. In all blocks, the location of the peripheral cue and the direction of the central target arrow were unrelated. Thus, in every block, the target pointed to the cued response box on 20 trials, yielding $40 \%$ of the trials with potential IOR effects. Furthermore, the arrow pointed to the uncued response box on 20 trials and did not appear on 10 trials (catch trials). The touch interface was individually calibrated for each participant, and a short practice session was given before data collection in each block. The order of the blocks was randomized for each participant, and short breaks were given between blocks. Errors were infrequent and were not analyzed.

Dependent measures were RT and MT. RT was the time from onset of the central cue to the registration of either a touchdown (for the ST and CT responses) or a lift-off (for the SM and CM responses) of the index finger on or from the touch screen. MT was 


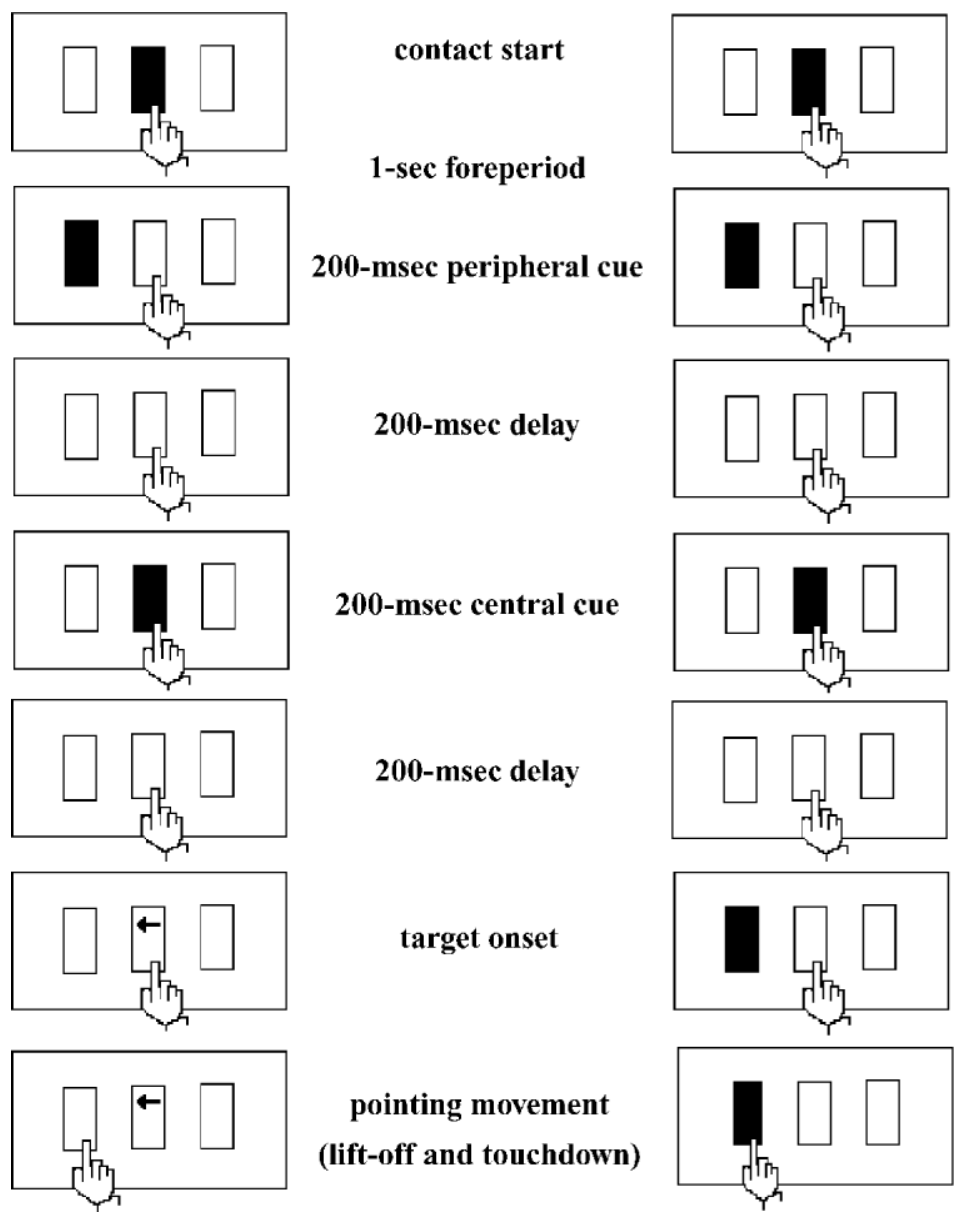

Figure 1. Schematic of display sequences in Experiment 1 (left) and Experiment 3 (right). Time runs from top to bottom.

the time from the registration of a lift-off to the registration of a touchdown on the touch screen (only for the SM and CM responses). ${ }^{2}$

To calculate IOR, we classified responses according to the convention of whether the target (the arrow) pointed to the cued or the uncued location. Note that in the ST and SM conditions, the responses were made to a specific location regardless of the direction of the arrow. Because of this, possible effects of spatial compatibility between target direction and response location were analyzed for the ST and SM responses. If IOR is a motor bias, than slower RTs should be found when the response was made to a cued location, regardless of the direction of the central arrow.

\section{Results and Discussion}

Of the responses, $4.4 \%$ were anticipations (mostly owing to the sensitivity of the touch screen to small finger movements on the start location during the foreperiod), $0.2 \%$ were procrastinations, and $1.2 \%$ were target misses. We did not record how these errors were distributed across conditions and, hence, did not analyze errors further. The remaining $94.2 \%$ of the data were analyzed as follows.

Consider first the RTs. The mean RTs for each of the nine blocks of response conditions are shown in Table 1
(Central Targets columns). A 4 (response: ST, CT, SM, or $\mathrm{CM}) \times 2$ (trial type: cued or uncued) analysis of variance (ANOVA) was used to analyze the data. For this analysis, we averaged across cue locations, target locations, and responding hands. There was a significant main effect of response $[F(3,42)=33.50, p<.001]$. Mean RTs for the ST, CT, SM, and CM responses were $473,523,371$, and $378 \mathrm{msec}$, respectively. Simple effects tests showed that all the responses differed reliably from one another $(p<.02)$, except SM and CM $(p>$ $.18)$. The main effect of trial type was not significant $[F(1,14)<2.5, p>.14]$, with the mean response times in cued $(413 \mathrm{msec})$ and uncued $(411 \mathrm{msec})$ trials being almost identical. Thus, there was no evidence of IOR. Also, the interaction between response and trial type was not significant $[F(3,42)<1]$, indicating that IOR was not present with any of the responses and ruling out the prediction that only movement responses would be sensitive to IOR.

In addition to RTs, the mean MTs for the two responses with hand transport are presented in the Central Targets columns of Table 2. A 2 (response: CM or SM) $\times 2$ (trial type: cued or uncued) ANOVA was used to an- 
Table 1

Reaction Times (RTs) in Experiment 1 (Central Targets) and Experiment 3 (Peripheral Targets)

\begin{tabular}{|c|c|c|c|c|c|c|c|c|c|c|c|c|}
\hline \multirow[b]{3}{*}{ Condition } & \multirow{3}{*}{$\begin{array}{c}\text { Active } \\
\text { Hand }\end{array}$} & \multirow{3}{*}{$\begin{array}{c}\text { Target } \\
\text { Position }\end{array}$} & \multicolumn{5}{|c|}{ Central Targets } & \multicolumn{5}{|c|}{ Peripheral Targets } \\
\hline & & & \multicolumn{2}{|c|}{ Cued } & \multicolumn{2}{|c|}{ Uncued } & \multirow[b]{2}{*}{ Difference } & \multicolumn{2}{|c|}{ Cued } & \multicolumn{2}{|c|}{ Uncued } & \multirow[b]{2}{*}{ Difference } \\
\hline & & & RT & $S D$ & RT & $S D$ & & RT & $S D$ & RT & $S D$ & \\
\hline \multirow[t]{2}{*}{ Simple touch } & $\mathrm{L}$ & $\mathrm{L}$ & 475 & 101 & 478 & 93 & -3 & 430 & 57 & 393 & 63 & 37 \\
\hline & $\mathrm{R}$ & $\mathrm{R}$ & 472 & 86 & 466 & 99 & 6 & 435 & 57 & 397 & 64 & 38 \\
\hline \multirow[t]{2}{*}{ Choice touch } & $\mathrm{L}$ & $\mathrm{L}$ & 515 & 111 & 516 & 102 & -1 & 468 & 140 & 451 & 148 & 19 \\
\hline & $\mathrm{R}$ & $\mathrm{R}$ & 539 & 102 & 521 & 100 & 18 & 476 & 122 & 454 & 118 & 22 \\
\hline \multirow[t]{4}{*}{ Simple movement } & $\mathrm{L}$ & $\mathrm{L}$ & 365 & 54 & 364 & 50 & 1 & 401 & 76 & 365 & 78 & 36 \\
\hline & $\mathrm{L}$ & $\mathrm{R}$ & 372 & 50 & 365 & 47 & 7 & 384 & 63 & 357 & 73 & 27 \\
\hline & $\mathrm{R}$ & $\mathrm{L}$ & 380 & 57 & 381 & 60 & -1 & 385 & 61 & 353 & 73 & 32 \\
\hline & $\mathrm{R}$ & $\mathrm{R}$ & 371 & 53 & 371 & 48 & 0 & 357 & 65 & 327 & 54 & 30 \\
\hline \multirow[t]{4}{*}{ Choice movement } & $\mathrm{L}$ & $\mathrm{L}$ & 376 & 43 & 378 & 48 & -2 & 346 & 60 & 313 & 44 & 33 \\
\hline & $\mathrm{L}$ & $\mathrm{R}$ & 376 & 43 & 378 & 48 & -2 & 361 & 67 & 329 & 52 & 32 \\
\hline & $\mathrm{R}$ & $\mathrm{L}$ & 381 & 49 & 382 & 45 & -1 & 361 & 70 & 354 & 79 & 7 \\
\hline & $\mathrm{R}$ & $\mathrm{R}$ & 384 & 43 & 369 & 44 & 15 & 323 & 61 & 328 & 67 & -5 \\
\hline
\end{tabular}

Note-L, left; R, right. Conditions are explained in the main text.

alyze these data. There was no significant main effect of response $[F(1,14)=2.95, p>.11]$, since the average MTs for SM and CM were 368 and $380 \mathrm{msec}$, respectively. The main effect of trial type also was not significant $[F(1,14)<1]$, with the average MTs in cued (374 $\mathrm{msec})$ and uncued $(373 \mathrm{msec})$ trials being almost identical. There was also no interaction between response and trial type $[F(1,14)<1]$.

Finally, the effect of spatial compatibility between arrow direction and response location on response speed was assessed in the two responses without directional choice (ST and SM). A 2 (response) $\times 2$ (compatibility) $\times 2$ (trial type) ANOVA on RTs indicated significant main effects of response $[F(1,14)=31.01, p<.001]$ and compatibility $[F(1,14)=18.87, p<.001]$, with all other $p$ values being $>.10$. RTs were $100 \mathrm{msec}$ shorter for the SM response (reflecting lift-off time) than for the ST response (reflecting touchdown time) and $19 \mathrm{msec}$ shorter in compatible than in incompatible responses. A 2 (compatibility) $\times 2$ (trial type) ANOVA on the MT data from the SM response alone showed no reliable effects, with all $p$ values being $>.51$.

Overall, there was no evidence from the present experiment that keypresses or guided pointing movements made in response to central targets are differentially sensitive to IOR. Of particular interest is the lack of IOR with CM responses, which is essentially the limb movement equivalent of the saccadic responses that have shown IOR in the studies by Abrams and Dobkin (1994), Abrams and Pratt (2000), and Taylor and Klein (2000). In the present study, however, the RTs for cued trials were only 2 msec longer, on average, than the RTs for uncued trials with guided pointing responses. Thus, the present results support the notion that motor IOR is specific to the oculomotor system.

However, it may also be the case, in the present experiment, that no IOR was found because eye movements to the peripheral locations had been made in response to the central target, but before the initiation of the manual responses. It may be that the inhibition asso- ciated with the manual response was essentially co-opted by faster occurring eye movements. This possibility was addressed in the second experiment.

\section{EXPERIMENT 2}

To add support to the earlier finding that IOR does not occur with peripheral cues and central targets demanding guided pointing responses, a control experiment is reported in which eye position was monitored to ensure that the participants remained fixated throughout. Only the $\mathrm{CM}$ condition of the first experiment was replicated.

\section{Method}

Participants. Seven participants from the Max Planck Institute for Psychological Research (Munich) volunteered to take part in the experiment. All the participants had normal or corrected vision. Their ages ranged from 18 to 32 years, and they responded with the right hand throughout. The participants were unaware of the hypotheses under investigation and were paid for their participation.

Apparatus. Targets for manual pointing were produced by illuminating light-emitting diodes (LEDs) inserted in a table. The table was $79 \mathrm{~cm}$ above ground and tilted $20^{\circ}$ out of the horizontal toward the participant. Paper circles (radius, $1.25 \mathrm{~mm}$ ) with embedded LEDs marked two pointing targets $10 \mathrm{~cm}$ on the left and right from a central fixation LED. Eye movements were recorded with the head-mounted Eyelink system (Sensomotoric Instruments), and movements of the right hand were recorded via an Optotrak system (Northern Digital), both using separate infrared cameras operating at $250 \mathrm{~Hz}$.

Procedure. The participants were asked to maintain central fixation throughout. For each trial, when eye fixation was within $2^{\circ}$ of a central yellow LED for $500 \mathrm{msec}$, a peripheral cue appeared for

Table 2

Movement Times (MTs, in Milliseconds) in Experiment 1 (Central Targets) and Experiment 3 (Peripheral Targets)

\begin{tabular}{cccccccc}
\hline & \multicolumn{3}{c}{ Central Targets } & & \multicolumn{3}{c}{ Peripheral Targets } \\
\cline { 2 - 4 } \cline { 6 - 8 } Condition & Cued & Uncued & Difference & & Cued & Uncued & Difference \\
\hline Simple move & 369 & 367 & 2 & & 315 & 319 & -4 \\
Choice move & 380 & 380 & 0 & & 332 & 340 & -8 \\
\hline
\end{tabular}

Note-Conditions are explained in the main text. 
$100 \mathrm{msec}$ in the left or right target circle. After a 400-msec interstimulus interval, the central LED was turned off for $100 \mathrm{msec}$ to direct attention back to fixation. Another $400 \mathrm{msec}$ after its reillumination, the yellow fixation LED turned either red or green, indicating that a movement of the right hand to the left or right target had to be made while fixation of the central LED had to be maintained. Thus, the SOA between the peripheral cue and the central target was $1,000 \mathrm{msec}$ and the task resembled the $\mathrm{CM}$ condition of Experiment 1.

Design. The four experimental conditions (left and right cues, followed by either congruent or incongruent central target instructions) were all repeated 20 times, resulting in one block of 80 trials per participant. The order of conditions was randomized.

Movement onset was the time at which the hand movement velocity exceeded $5 \%$ of its peak velocity, and movement offset was the time at which it dropped below $5 \%$ of peak velocity again. RT was defined as the time between target onset and hand movement onset, and MT as the time between hand movement onset and offset. For fixation control, saccade onset was defined as the moment at which eye movement velocity exceeded $35 \mathrm{deg} / \mathrm{sec}$ and eye movement acceleration exceeded 3,900 deg/ $/ \mathrm{sec}^{2}$.

\section{Results and Discussion}

Eye fixation errors were made on fewer than $10 \%$ of the trials and were not analyzed further. Manual performance in the remaining trials was analyzed with paired $t$ tests to assess the effect of trial type, averaging across side of response. No reliable difference was found between mean RTs for cued $(423 \mathrm{msec})$ and uncued $(415 \mathrm{msec})$ trials $[t(6)<1]$. The mean MTs also yielded no significant differences between cued $(565 \mathrm{msec})$ and uncued $(559 \mathrm{msec})$ trials $[t(6)<1]$.

Overall, the data from this control experiment support the earlier finding of no IOR with guided pointing responses to central targets. In addition, the present results show that this result is obtained in the absence of eye movements and with a different apparatus. This further strengthens the notion that motor IOR is oculomotor specific and occurs only when eye movements are involved.

\section{EXPERIMENT 3}

The first two experiments failed to reveal IOR with goal-directed manual responses to central targets. This result was obtained twice, first with a touch-sensitive CRT and then with LED cues and on-line movement registration. The convergence of results obtained with different methods and protocols lends strength to our conclusion that none of the manual responses tested were sensitive to IOR. However, it is also possible that some aspect of our experimental methodology or apparatus negated any possible IOR effects. To test this possibility, in the third experiment, the same types of responses and the same equipment were used as in the first experiment, but this time the responses were made to peripheral targets.

Assuming that visual-processing IOR is not response specific (i.e., the inhibited visual processing of a peripheral target occurs regardless of a keypress, a pointing, or a saccadic response), IOR should be found with the four types of manual responses initially tested in Experi- ment 1 . Indeed, there is considerable evidence to support this prediction. Of course, there are numerous examples of IOR with peripheral targets and keypress responses (e.g., Maylor \& Hockey, 1985; Posner \& Cohen, 1984). In addition, there are also examples of IOR to peripheral targets with guided computer mouse movements (Briand, Larrison, \& Sereno, 2000; Tanaka \& Shimojo, 1996) and guided reaching responses (Howard, Lupiáñez, \& Tipper, 1999). It is worth noting, however, that these previous studies used the type of response that would be functionally equivalent to our $\mathrm{CM}$ condition and did not compare the IOR effects found between ST, CT, SM, and CM responses. Thus, although we expected IOR with all four responses, it was not clear that all four responses would yield equivalent magnitudes of IOR.

\section{Method}

Participants. Fifteen members from the human participants pool of the Psychology Department at the University of Munich, including one of the authors, participated in the experiment. Eleven participants were female; 10 participants were right-handed, 4 were left-handed, and 1 was ambidextrous, according to self-report. Their ages ranged from 22 to 45 years (average, 32 years). All the participants had normal or corrected vision. The naive participants received DM $12 / \mathrm{h}$.

Apparatus, Procedure, and Design. The apparatus, procedures, and design were identical to those in the first experiment, except for the following modifications of the display sequence. Instead of the target being a central arrow, the area encompassed by one of the peripheral boxes changed from black to green to indicate the target. The participants were to make their response to either the preassigned target box (ST and SM) or the location indicated by the green box (CT and $\mathrm{CM}$ ). This peripheral target remained visible until either a touchdown had been registered or the maximum trial duration had elapsed. In catch trials, neither peripheral box was changed to green. The same responses as those in Experiment 1 were used.

\section{Results and Discussion}

Of the responses, $5.5 \%$ were anticipations (again, mostly owing to the sensitivity of the touch screen to changes in finger pressure on the screen during the foreperiod), $0.6 \%$ were procrastinations, and $2.3 \%$ were target misses. We did not keep a record of how these error types were distributed across conditions; hence, the errors were not further analyzed. The remaining $91.6 \%$ of the data were analyzed as before.

Consider first the RTs. The mean RTs for each of the nine blocks of responses conditions can be seen in the right portion of Table 1. A 4 (response: ST, CT, SM, or CM) $\times 2$ (trial type: cued or uncued) ANOVA was again used to analyze the data. There was a significant main effect of response $[F(3,42)=9.56, p<.001]$. Average RTs for ST, CT, SM, and CM responses were 414, 462, 366, and $339 \mathrm{msec}$, respectively. Simple effects tests showed that all the responses differed reliably from one another $(p<.02)$, except ST and SM $(p>.19)$. The main effect of trial type was also significant $[F(1,14)=91.99, p<$ $.001]$, with longer RTs in cued (410 msec) than in uncued $(388 \mathrm{msec})$ trials, indicating the presence of IOR. The interaction between response and trial type was also sig- 
nificant $[F(3,42)=3.22, p<.05]$. The size of the IOR effects for ST, CT, SM, and CM responses was 38, 21, 31, and $17 \mathrm{msec}$, respectively. Simple effects tests showed that this IOR effect was reliable within each response $(p<.01)$. Furthermore, the IOR effect was reliably larger for SM responses than for CM responses $(p<.05)$.

Consider now MTs. The mean MTs for the SM and CM responses are shown in the right portion of Table 2. There was a marginally significant main effect of response $[F(1,14)=3.44, p<.09]$. MTs were slightly slower for the CM response (336 $\mathrm{msec})$ than for the SM response $(317 \mathrm{msec})$, presumably owing to advance knowledge of the response location in the SM response. Interestingly, the main effect of trial type was significant $[F(1,14)=4.43, p=.05]$, showing shorter MTs toward cued locations (324 msec) than toward uncued locations $(330 \mathrm{msec})$. Thus, responses to inhibited locations actually yielded reliably shorter MTs. This novel finding will be further evaluated in the General Discussion section. The interaction between response and trial type was, however, not significant $[F(1,14)=0.92, p>.35]$.

Finally, the effect of spatial compatibility between target position and response location on response speed was again assessed for the two responses without directional choice (ST and SM). A 2 (response) $\times 2$ (compatibility) $\times 2$ (trial type) ANOVA on RT indicated significant main effects of response $[F(1,14)=35.62, p<.001]$, of compatibility $[F(1,14)=40.70, p<.001]$, and of trial type $[F(1,14)=57.63, p<.001]$. RTs were $46 \mathrm{msec}$ shorter for SM response (reflecting lift-off time) than for the ST response (reflecting touchdown time), $58 \mathrm{msec}$ shorter for compatible than for incompatible responses, and $34 \mathrm{msec}$ shorter for uncued than for cued responses. The only reliable interaction was between response and compatibility $[F(1,14)=6.05, p<.05]$, with all other $p$ values being $>.10$. The compatibility effect was $15 \mathrm{msec}$ larger for the ST response than for the SM response. A 2 (compatibility) $\times 2$ (trial type) ANOVA on the MT data from the SM response alone indicated a marginally reliable effect of compatibility $[F(1,14)=4.47, p=.05]$, with all other $p$ values being $>.19$. Movements toward the target were completed $5 \mathrm{msec}$ faster than incompatible movements.

As was expected, IOR was found with all four types of manual responses. This indicates that the lack of IOR found in the previous two experiments were not due to the particularities of the displays or timing of the events. Interestingly, IOR was found in the SM condition even when the movement was made to the location opposite to that of the target. This is strong evidence that a visualprocessing form of IOR does occur when there are no eye movements, since the inhibited visual processing of the cue slowed down responses made in the opposite direction. Moreover, it is also evidence for the oculomotorspecific motor form of IOR, since a general motor form of IOR would have slowed responses to the movement location, and not to the location where the peripheral target appeared.

\section{GENERAL DISCUSSION}

Taylor and Klein (2000) postulated that there are two forms of IOR. One form, visual IOR, slows visual processing and occurs when eye movements are not made. The other form, motor IOR, slows motor responses and occurs when eye movements are made. Although Taylor and Klein (2000) were able to dissociate these two forms of IOR, their extensive study did not have the conditions to determine whether motor IOR is specific to oculomotor responses or whether it occurs for any type of spatially directed responses. They did, however, note that the critical test for this determination would be to examine manual pointing movements made in response to central targets. This was the major focus of the present experiments, in which four types of responses (ST, CT, SM, and CM) were made in response to both central (Experiments 1 and 2) and peripheral (Experiment 3) targets.

The major finding from these experiments was that manual pointing responses did not yield IOR with central targets. Following Taylor and Klein's (2000) framework for IOR, this indicates that motor IOR is limited to the oculomotor system and is not a general motor effect that would inhibit any spatially directed response. This conclusion has several implications. First, it adds support to the Taylor and Klein's (2000) notion that a single form of IOR cannot account for the presence of IOR across all conditions. This is not a trivial point, since previous researchers have often explained the inhibitory effects found in their experiments solely in terms of either an attentional (i.e., visual processing) effect (e.g., Pratt, Kingstone, \& Khoe, 1997) or a motor effect (e.g., Taylor $\&$ Klein, 1998). Second, and the flip side of the coin to the first implication, the present conclusions support the contention that the general notion of IOR may best be conceived of as a combination of attentional and oculomotor effects (Kingstone \& Pratt, 1999; Klein, 2000). Thus, depending on the experimental methodology, it may be possible to activate one form of IOR or the other, or both simultaneously. Third, given that manual pointing movements involve the SC, it seems unlikely that the $\mathrm{SC}$ is the sole locus of IOR (as has been suggested by Dorris et al., 2002; Klein, 2000; see, also, Fuentes, Vivas, \& Humphreys, 1999, for an example of IOR effects in nonspatial dimensions). Fourth, an important course for future research will be to examine the similarities and differences between the two forms of IOR, to determine whether a single process or separate processes underlie the inhibition. In fact, some work regarding this issue is already at hand, since Abrams and Pratt (2000) have shown that oculomotor IOR is coded in retinal coordinates while visual-processing (i.e., attentional) IOR is encoded in environmental coordinates.

Overall, the results from the three experiments are consistent with the framework laid out by Taylor and Klein (2000). For example, they postulated that the visualprocessing form of IOR will occur when the eyes are held at fixation and peripheral targets are used. Thus, this 
form of inhibition should affect all types of manual responses, because the inhibition occurs during the visual processing of the peripheral target before any specific response is planned. This was found in Experiment 3, where ST, CT, SM, and CM responses all showed IOR. As was noted earlier, the fact that IOR was found for antimovements (trials in the SM condition in which the pointing movement was made in the direction opposite to the peripheral target) further supports the existence of a nonmotor form of IOR. Otherwise, the inhibition for such antimovements would not have been found. The lack of IOR with the keypress type responses to central targets is also consistent with Taylor and Klein's (2000) notion of visual-processing IOR, since in the absence of motor IOR owing to eye movements, there are no peripheral targets to be visually processed and, hence, no inhibition.

One possible complication to the present conclusions is that the time course of IOR may have differed in the first and the third experiments. Thus, although the same cues, event sequence, and responses were used in the two experiments, the absence of IOR in the first experiment and the presence of IOR in the third may have been obtained simply because IOR occurred at a later time with central targets. Indeed, Lupiáñez and his colleagues (e.g., Lupiáñez, Milán, Tornay, Madrid, \& Tudela, 1997; Lupiáñez, Milliken, Solano, Weaver, \& Tipper, 2001) have shown that IOR begins later and ends sooner as task difficulty increases. However, there are reasons to question such a time course explanation of the present findings. First, the SOAs used with the central targets in Experiments 1 and 2 (800 and 1,000 msec, respectively) are the SOAs for which Lupiáñez and co-workers found IOR with all of their tasks (simple detection, color detection, go-no-go, and shape discrimination). Second, there is evidence that the presence of a fixation cue tends to negate time course effects (following the notion that IOR begins when attention is withdrawn from the cued peripheral location-i.e., when the central fixation cue is presented). For example, using fixation cues, Wright and Richard (1996) found IOR at very short (200 and $400 \mathrm{msec}$ ) SOAs. Third, the RTs for the CM responses in the first experiment $(378 \mathrm{msec})$ were, in fact, moderately shorter than those found in the third experiment $(414 \mathrm{msec})$, suggesting that the pointing responses were equally difficult in both the central and the peripheral target experiments. Finally, the SOA used in the present experiments was in the same range as the single SOA used by Taylor and Klein (2000). Indeed, they also used fixation cues that presumably mediated the time course effect on IOR of the different responses.

An unexpected aspect of the present results is the finding of marginally shorter MTs toward cued than toward uncued locations in Experiment 3 (a similar effect was noted by Howard et al., 1999). Thus, we found a tradeoff between RTs and MTs in responses with overt movements, with RTs showing inhibition, whereas MTs showed facilitation from cuing. Note that this effect of trial type is independent of the compatibility between the target lo- cation and the response location. For example, a response to the left box was considered spatially compatible both when the cue had appeared at the left box (cued trial) and when the cue had appeared at the right box (uncued trial). This observation of facilitated MT toward an inhibited location (as indicated by the RT effect) should be replicated with better control of error probabilities. If the effect replicates in future studies, it further argues against the notion of a general motor IOR, because it demonstrates selective inhibition of response initiation but facilitated response execution toward cued locations. Interestingly, Howard et al. also concluded that there was not a general form of motor IOR in the reaching task, after using a methodology that included peripheral cues and targets. In this case, RTs were slowed to cued targets (presumably reflecting the visual-processing form of IOR), but the trajectories of the pointing responses were not affected (presumably reflecting the lack of a general motor form of IOR).

In summary, the results of the present experiments are consistent with Taylor and Klein's (2000) notion that there are two forms of IOR; a visual-processing form and a motor form. Importantly, the present results clearly show that the motor form of IOR does not generalize to all types of spatially localized responses, since no IOR was found with manual pointing responses to central targets. Overall, there seems to be strong evidence to suggest that the visual-processing form of IOR occurs when eye movements are not made (or planned) and that the motor form of IOR is specific to the oculomotor system and occurs when eye movements are made (or planned). The challenge of future research will be to understand the relationship between these two types of IOR.

\section{REFERENCES}

Aвrams, R. A., \& Dobkin, R. S. (1994). Inhibition of return: Effects of attentional cuing on eye movement latencies. Journal of Experimental Psychology: Human Perception \& Performance, 20, 467-477.

Abrams, R. A., \& Pratt, J. (2000). Oculocentric coding of inhibited eye movements to recently attended locations. Journal of Experimental Psychology: Human Perception \& Performance, 26, 776-788.

Bekkering, H., Pratt, J., \& Abrams, R. A. (1996). The gap effect for eye and hand movements. Perception \& Psychophysics, 58, 628-635.

Briand, K. A., Larrison, A. L., \& Sereno, A. B. (2000). Inhibition of return in manual and saccadic response systems. Perception \& Psychophysics, 62, 1512-1524.

Dorris, M. C., Klein, R. M., Everling, S., \& Munoz, D. P. (2002). Contribution of the primate superior colliculus to inhibition of return. Journal of Cognitive Neuroscience, 14, 1256-1263.

Fischer, M. H. (1997). Attention allocation during manual movement preparation and execution. European Journal of Cognitive Psychology, 9, 17-51.

Fuentes, L. J., Vivas, A. B., \& Humphreys, G. W. (1999). Inhibitory tagging of stimulus properties in inhibition of return: Effects on semantic priming and flanker interference. Quarterly Journal of Experimental Psychology, 52A, 149-164.

Howard, L. A., Lupiáñez, J., \& Tipper, S. P. (1999). Inhibition of return in a selective reaching task: An investigation of reference frames. Journal of General Psychology, 126, 421-442.

Kingstone, A., \& Pratt, J. (1999). Inhibition of return is composed of attentional and oculomotor processes. Perception \& Psychophysics, 61, 1046-1054. 
KLEIN, R. M. (2000). Inhibition of return. Trends in Cognitive Sciences, 4, 138-147.

Klein, R. [M.], \& TAYloR, T. L. (1994). Categories of cognitive inhibition with reference to attention. In D. Dagenbach \& T. H. Carr (Eds.), Inhibitory processes in attention, memory, and language (pp. 113-150). New York: Academic Press.

Lupiáñez, J., Milán, E. G., Tornay, F. J., Madrid, E., \& Tudela, P. (1997). Does IOR occur in discrimination tasks? Yes, it does, but later. Perception \& Psychophysics, 59, 1241-1254.

Lupiáñez, J., Milliken, B., Solano, C., Weaver, B., \& Tipper, S. (2001). On the strategic modulation of the time course of facilitation and inhibition of return. Quarterly Journal of Experimental Psychology, 54A, 753-773.

MAYLOR, E. A., \& Hockey, R. (1985). Inhibitory component of externally controlled covert orienting in visual space. Journal of Experimental Psychology: Human Perception \& Performance, 11, 777-787.

Posner, M. I., \& Cohen, Y. (1984). Components of visual orienting. In H. Bouma \& D. G. Bouwhuis (Eds.), Attention and performance X: Control of language processes (pp. 531-556). Hillsdale, NJ: Erlbaum.

Pratt, J., Kingstone, A., \& KhoE, W. (1997). Inhibition of return in location- and identity-based choice of decision tasks. Perception \& Psychophysics, 59, 964-971.

Rafal, R. D., Calabresi, P. A., Brennan, C. W., \& Sciolto, T. K. (1989). Saccade preparation inhibits reorienting to recently attended locations. Journal of Experimental Psychology: Human Perception \& Performance, 15, 673-685.

SAPIr, A., Soroker, N., Berger, A., \& Henik, A. (1999). Inhibition of return in spatial attention: Direct evidence for collicular generation. Nature Neuroscience, 2, 1053-1054.

Stuphorn, V., Hoffmann, K. P., \& Miller, L. E. (1999). Correlation of primate superior colliculus and reticular formation discharge with proximal limb muscle activity. Journal of Neurophysiology, 81, 1978-1982.

TAnaKa, Y., \& Shimojo, S. H. (1996). Vision, attention, and action: Inhibition and facilitation in sensory-motor links revealed by the reaction time and the line motion. Vision Research, 36, 2125-2140.
TAYlor, T. L., \& KLEIN, R. M. (1998). On the causes and effects of inhibition of return. Psychonomic Bulletin \& Review, 5, 625-643.

TAYLOR, T. L., \& KLEIN, R. M. (2000). Visual and motor effects in inhibition of return. Journal of Experimental Psychology: Human Perception \& Performance, 26, 1639-1656.

TipPer, S. P., Driver, J., \& WeAver, B. (1991). Object-centred inhibition of return of visual attention. Quarterly Journal of Experimental Psychology, 43A, 289-298.

WERnER, W. (1993). Neurons in the primate superior colliculus are active before and during arm movements to visual targets. European Journal of Neuroscience, 5, 333-340.

WRIGHT, R. D., \& RICHARD, C. M. (1996). Inhibition-of-return at multiple locations in visual space. Canadian Journal of Experimental Psychology, 50, 324-327.

\section{NOTES}

1. To distinguish between response instruction and response location, we adopt the following terminology throughout this manuscript: Responseirrelevant events are cues, response-relevant events are targets, and the locations to which responses are directed are response locations.

2. Note that the definition of RT differs between the responses with versus without hand movements toward peripheral targets: RT includes the time to flex the index finger for the ST and CT responses. RTs would be more comparable to those for the SM and CM responses if participants were asked to just lift their fingers for the ST and CT responses, but it is not clear whether this RT would still include movement planningfor example, toward some subsequent resting location (see Fischer, 1997 , for a similar argument and for empirical comparisons). The relatively long RTs obtained in the touch responses (see below) may also be due to force control requirements while the hand is held above the display. We thank an anonymous reviewer for pointing this out.

(Manuscript received December 31, 2000; revision accepted for publication July 31,2002 .) 